\title{
Changes in portlandite morphology with solvent composition: Atomistic simulations and experiment
}

\author{
Sandra Galmarini *, Anne Aimable ${ }^{1}$, Nicolas Ruffray, Paul Bowen \\ Powder Technology Laboratory (LTP), Ecole Polytechnique Fédérale de Lausanne (EPFL), CH-1015 Lausanne, Switzerland
}

\section{A R T I C L E I N F O}

Article history:

Received 8 November 2010

Accepted 17 April 2011

\section{Keywords:}

$\mathrm{Ca}(\mathrm{OH})_{2}$

Image analysis

Microstructure

Modeling

\begin{abstract}
A B S T R A C T
Experimental work has been done to determine changes in the particle shape of portlandite grown in the presence of different ions. To quantify the experimentally observed changes in morphology a new analysis tool was developed, allowing the calculation of the relative surface energies of the crystal facets. The observed morphology in the presence of chlorides and nitrates was facetted particles of a similar shape, the addition of sulfates leads to hexagonal platelet morphology and the addition of silicates leads to the formation of large irregular aggregates. In addition to the experimental work, the surfaces of portlandite were studied with atomistic simulation techniques. The empirical force field used has first been validated. The equilibrium morphology of portlandite in vacuum and in water was then calculated. The results indicate that the presence of water stabilizes the [20.3] surface and changes the morphology. This is consistent with the experimental observation of [20.3] surfaces.
\end{abstract}

(c) 2011 Elsevier Ltd. All rights reserved.

\section{Introduction}

The purpose of this work is to understand changes in portlandite $(\mathrm{CH})$ morphology with the changing chemical environment of the growing crystal as encountered in cementitious systems. If these changes in morphology can be understood, they may be controlled and modified. As portlandite is a major component of hydrated cementitious systems and concrete, a change in morphology might modify the durability and may allow the fine tuning of the physical properties of concrete structures. However while the crystal structure of portlandite is well known [1-3], not much experimental work has been done to study the growth mechanisms. Work from Gallucci and Scrivener [4] shows a change in portlandite morphology from large clusters to small dispersed and sometimes hexagonal platelets on addition of gypsum ( $C \$$ ) and tricalcium aluminate $\left(C_{3} A\right)$. This indicates that the interaction of portlandite surfaces and surface steps with gypsum and $\mathrm{C}_{3} \mathrm{~A}$ dissolution products might give some insight into the working mechanisms of the observed change in morphology. Harutyunyan et al., used soft X-ray techniques to observe the growth of $\mathrm{CH}$ near cement particles in a $\mathrm{CH}$ and $\mathrm{C} \$$ saturated solution [5]. The observed portlandite particles had a hexagonal platelet morphology with a aspect ratio of 2.5 to 2.7. The

\footnotetext{
* Corresponding author.

E-mail address: sandra.galmarini@epfl.ch (S. Galmarini).

1 Science des Procédés Céramiques et de Traitements de Surface (SPCTS), Ecole Nationale Supérieure de Céramique Industrielle (ENSCI), 87068 Limoges Cedex, France.
}

surface energy of the particles was estimated to be approximately $0.114 \mathrm{~J} / \mathrm{m}^{2}$. Berger and McGregor, used optical microscopy to observe the influence of different additives on the morphology of $\mathrm{CH}$ formed upon hydration of tricalcium silicate $\left(\mathrm{C}_{3} \mathrm{~S}\right)$ [4]. They observed that the addition of nitrates and chlorides led to the formation of rod-like instead of platelet-like morphologies whereas the addition of sulfates reinforced the platelet morphology. The addition of hydroxides led to more but smaller particles.

So far the studies were carried out in complex systems with a large number of different ions present in the solvent surrounding the growing portlandite crystals. In these systems it is difficult to assess how the observed changes in morphology were brought about and which species in solution were responsible for it. Consequently experimental work on pure model systems has been carried out. Portlandite particles were produced by coprecipitation of a calcium and a hydroxide salt. Chloride, nitrate, sulfate and silicate salts were then added to different batches to observe their influence on the morphology of portlandite.

To understand the changes in portlandite morphology and to characterize portlandite surfaces, atomistic simulation techniques, namely classical energy minimization and classical molecular dynamics, have been employed. These techniques use empirical force fields to describe the forces between the atoms and the choice and validation of the employed force field is described. Using these results the equilibrium or Wulff shape of portlandite in vacuum and in water was calculated.

Apart from several articles using portlandite for the atomistic potential development and validation [6-8] there is, to our knowledge, only one group who have used atomistic simulation to study 
portlandite and portlandite surfaces: Kalinichev et al. used classical molecular dynamics to look at the structure of the water above the surface and to estimate the extent of chloride binding at portlandite surfaces $[9,10]$. The authors restricted their study to the [00.1] surface and did not attempt to estimate the surface energy.

\section{Materials and methods}

\subsection{Materials}

The following chemical products were used as received: calcium chloride $\mathrm{CaCl}_{2}, 2 \mathrm{H}_{2} \mathrm{O}$, calcium nitrate $\mathrm{Ca}\left(\mathrm{NO}_{3}\right)_{2}, 4 \mathrm{H}_{2} \mathrm{O}$, sodium chloride $\mathrm{NaCl}$, sodium sulfate $\mathrm{Na}_{2} \mathrm{SO}_{4}$ (Merck, quality grade GR for analysis), and a sodium hydroxide $50 \mathrm{wt}$.\% solution (Acros Organics, extra pure). Stock solutions at $1.0 \mathrm{M}$ were prepared by diluting the desired amount of reagent with ultrapure water. These solutions were then filtered at $0.2 \mu \mathrm{m}$ to eliminate dust. The stock solutions were stored in closed vessels to avoid further contamination, and used within a few days to avoid carbonation.

\subsection{Precipitation procedure}

The reactive solutions were prepared just before the precipitation by diluting the stock solutions with ultrapure water. Sample B was obtained by mixing $\mathrm{CaCl}_{2} 0.2 \mathrm{M}$ with $\mathrm{NaOH} 0.4 \mathrm{M}$. In sample $\mathrm{BC}$ and sample BS, respectively $0.1 \mathrm{M}$ of $\mathrm{NaCl}$ and $0.1 \mathrm{M}$ of $\mathrm{Na}_{2} \mathrm{SO}_{4}$ were added to both solutions. Sample BN was obtained by mixing $\mathrm{Ca}\left(\mathrm{NO}_{3}\right)_{2}$ $0.2 \mathrm{M}$ - instead of $\mathrm{CaCl}_{2}$ - with $\mathrm{NaOH} 0.4 \mathrm{M}$. The nominal concentrations of the different ions after mixing are summarized in Table 1.

The mixing was done by hand, by adding rapidly ( $5 \mathrm{~s}) 100 \mathrm{ml}$ of the calcium solution to $100 \mathrm{ml}$ of the sodium hydroxide solution in a $250 \mathrm{ml}$ glass beaker. A high stirring rate (200 to $500 \mathrm{rpm}$ with a magnetic stirrer) was maintained during $5 \mathrm{~min}$. Then the precipitation mixture was placed on rolling bars $(60 \mathrm{rpm})$ for mild stirring during one hour. The precipitate was finally collected by filtration using $0.2 \mu \mathrm{m}$ filters, washed with $100 \mathrm{ml}$ ultrapure water, and dried for $24 \mathrm{~h}$ at $60{ }^{\circ} \mathrm{C}$.

\subsection{Characterization methods}

The powder morphologies were analyzed by scanning electron microscopy (SEM, Philips XL 30 FEG microscope). The SEM samples were prepared by dispersing the powder in ethanol. The suspension was sonicated for $10 \mathrm{~min}$ in an ultrasonic bath, and one drop of the suspension was then deposited on an aluminum support and dried in air.

The phase identification of the precipitates was made with X-ray powder diffraction (XRD, Philips X'Pert diffractometer, $\mathrm{Cu}-\mathrm{K} \alpha$ radiation).

The particle size distribution (PSD) was collected using a laser diffraction method (Malvern Mastersizer S). The particles were suspended at $1 \mathrm{wt} . \%$ in a saturated $\mathrm{Ca}(\mathrm{OH})_{2}$ solution with poly(acrylic) acid $\mathrm{M}_{\mathrm{w}} 2000$ (0.1 wt.\%), to avoid dissolution during measurement.

Table 1

Nominal concentration of the different ions present in the precipitation mixture.

\begin{tabular}{lllllll}
\hline \multirow{2}{*}{ Sample } & \multicolumn{6}{l}{ Concentration after mixing $[\mathrm{mol} / \mathrm{l}]$} \\
\cline { 2 - 7 } & {$\left[\mathrm{Ca}^{2+}\right]$} & {$\left[\mathrm{HO}^{-}\right]$} & {$\left[\mathrm{Cl}^{-}\right]$} & {$\left[\mathrm{NO}_{3}^{-}\right]$} & {$\left[\mathrm{SO}_{4}^{2-}\right]$} & {$\left[\mathrm{SiO}_{3}^{2-}\right]$} \\
\hline B & 0.1 & 0.2 & 0.2 & - & - & - \\
BC & 0.1 & 0.2 & 0.3 & - & - & - \\
BN & 0.1 & 0.2 & - & 0.2 & - & - \\
BS & 0.1 & 0.2 & 0.2 & - & 0.1 & - \\
BS02 & 0.1 & 0.2 & 0.2 & - & 0.02 & - \\
BSi & 0.1 & 0.2 & 0.2 & - & - & 0.001
\end{tabular}

Thermogravimetric curves (TGA, Mettler TGA/DSC/TMA analyzer) were collected from room temperature to $600{ }^{\circ} \mathrm{C}$ under flowing air at a heating rate of $10^{\circ} \mathrm{C} / \mathrm{min}$.

\subsection{Quantification of morphological changes}

In order to compare the influence of different ions a more quantitative description of the changes in morphology is needed. Therefore a new analysis tool to extract the exact shape of observed facetted particles of regular shape was developed. The first step of the analysis was to digitalize the observed SEM projection of a portlandite particle by recording the positions of the corners of the shape. This was done manually. The corner coordinates were then read into an adapted version of the Wulffman program [11]. Wulffman is capable of calculating the equilibrium morphology for a system with known crystallographic symmetry and relative surface energies. The relative surface energies determine the distance of the corresponding facets from the centroid of the shape. Thus any facetted regular morphology can be reproduced with Wulffman, irrespective of whether or not the observed morphology corresponds to an equilibrium morphology and the relative surface energies of the shape have a physical meaning.

The Wulffman program was expanded as to be able to fit the projection angles and relative surface energies of the calculated particle shape to the experimentally observed shape. In order to compare two shapes, the facets of the two shapes have to be sorted the same way to ensure the right facets are compared with each other. The first facet in the sorting order is the facet furthest from the centroid of the total shape. The other facets are sorted in a clockwise manner. The final fitting function which was minimized to fit the calculated to the experimentally observed shape takes into account the relative projection area and position of the different facets as well as their shape (Eqs. (1) and (2)).

$\frac{1}{N_{\text {faces }}} \sum_{i}^{N_{\text {faces }}}\left[\alpha\left(\Theta_{i}-\Theta_{i}^{\text {exp }}\right)^{2}+\beta\left(\frac{r_{i}^{2}}{a_{\text {tot }}}-\frac{\left(r_{i}^{\text {exp }}\right)^{2}}{a_{\text {tot }}^{\text {exp }}}\right)^{2}+\gamma\left(\frac{a_{i}}{a_{\text {tot }}}-\frac{a_{i}^{\text {exp }}}{a_{\text {tot }}^{\text {exp }}}\right)^{2}+\delta F_{i}^{\text {shape }}\right]$

$F_{i}^{\text {shape }}=\frac{1}{N_{\text {corner }}^{i}} \sum_{j}^{N_{\text {corner }}^{i}}\left[\kappa\left(\psi_{j}^{i}-\psi_{j}^{i, \exp }\right)^{2}+\lambda\left|\frac{\left(l_{j}^{i}\right)^{2}}{a_{i}}-\frac{\left(l_{j}^{i, \exp }\right)^{2}}{a_{i}^{\exp }}\right|\right]$

where $\mathrm{N}_{\text {faces }}$ is the number of facets of the shape, $\mathrm{r}_{\mathrm{i}}$ is the position of the centroid of facet i relative to the centroid of the total shape, $\Theta_{i}$ is the angle between $r_{i}$ and $r_{1}, a_{i}$ is the area of facet $i, a_{\text {tot }}$ is the total area $\mathrm{N}_{\text {corner }}^{\mathrm{i}}$ is the number of corners of facet $\mathrm{i}, \mathrm{l}_{\mathrm{j}}^{\mathrm{i}}$ is edge $\mathrm{j}$ of facet $\mathrm{i}$ and $\Psi^{\mathrm{i}} \mathrm{j}$ is the angle between edge $\mathrm{j}$ and edge $\mathrm{j}+1$ of facet $\mathrm{i}$ (see Fig. 1 ). $\alpha, \beta, \gamma, \delta, \kappa$ and $\lambda$ are weighting parameters which were set to $\alpha=\beta=\gamma=\delta=0.25$ and $\kappa=\lambda=0.5$.

The parameters changed during the fitting are the relative surface energies, and the projection angles. The fitting was done via the simplex minimization method [12] of the GNU Scientific Library [13].

\subsection{Atomistic simulation}

All atomistic simulations done on portlandite were done with classical potentials. Both static energy minimization and molecular dynamics calculations were carried out. The same potential set was used for both methods. It has been chosen for its good performance for both the water and the inorganic components when compared to other force fields [14]. The following subsections describe the potential and the computational methods used in more detail.

\subsubsection{Potential}

The potential used for the inorganic materials was the potential developed by Freeman et al. [6]. It is an adaption of an earlier force field 

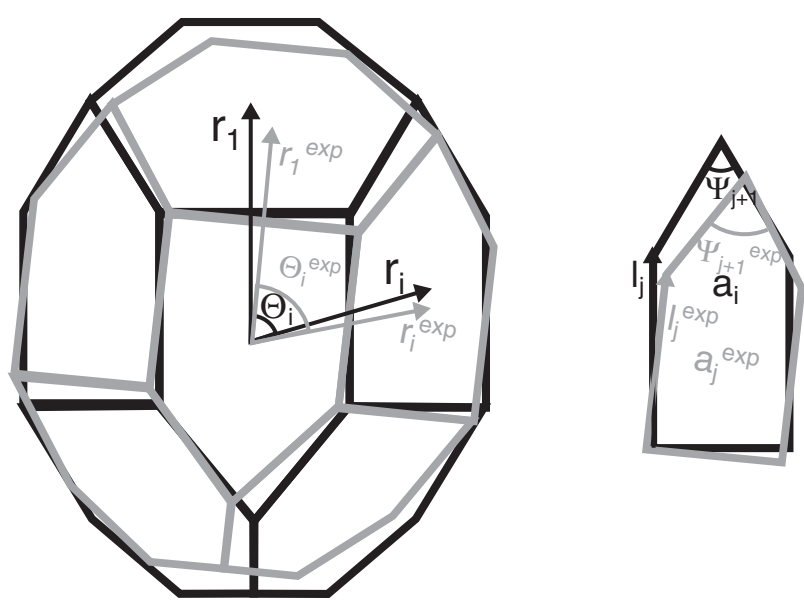

Fig. 1. Schematic view of parameters used for the fitting between the calculated and the experimentally observed shape.

$[7,15,16]$. The earlier force field $[7,15,16]$ has been shown repeatedly to give good results for the description of inorganic oxide crystals [7,1719]. Freeman et al. have shown that with a slight adaption the force field can be used in connection with rigid molecule water force fields. The resulting potential is a pair potential expressing the interaction $\left(V_{i j}\right)$ between two atoms of a certain type (atoms $i$ and $j$ ) as a function of the distance $\left(\mathrm{r}_{\mathrm{ij}}\right)$ between those two atoms. It takes into account coulombic (first term in Eq. (3)) and both repulsive and attractive short-range contributions (second and third term of Eq. (3) respectively).

$V_{i j}\left(r_{i j}\right)=V_{\text {coulombe }}+V_{\text {disp }}=\frac{q_{i} q_{j}}{r_{i j}}+A_{i j} \cdot e^{-\frac{r_{i j}}{\rho_{i j}}}-\frac{C_{i j}}{r_{i j}^{6}}$

where $\mathrm{q}$ is the charge of the respective ion and $\mathrm{A}_{\mathrm{ij}}, \rho_{\mathrm{ij}}$ and $\mathrm{C}_{\mathrm{ij}}$ are constants evaluated by fitting resulting properties to experimental data such as the lattice and elastic constants. In addition a Morse potential (Eq. (4), D, $\alpha$, and $r_{0}$ are fitted constants) describes the bond between the oxygen and the hydrogen of the hydroxyl group.

$V_{i j}\left(r_{i j}\right)=D\left[1-e^{-a\left(r_{i j}-r_{0}\right)}\right]^{2}-D$

The potential used to describe the water was the Tip4P/2005 potential [20], which is a rigid molecule water force field that yields very good results for water calculations. It reproduces a large portion of the phase diagram for water with only a slight shift in temperature [20]. The rigid water molecules are composed of four interaction sites: two positively charged hydrogen sites, one negatively charged oxygen site and a neutral Lennard-Jones interaction site (Eq. (5), $\varepsilon$ and $\sigma$ are fitted parameters) which is coplanar to the hydrogen and oxygen sites and located on the bisector of the $\mathrm{H}-\mathrm{O}-\mathrm{H}$ angle [20].

$V_{i j}\left(r_{i j}\right)=4 \varepsilon\left[\left(\frac{\sigma}{r_{i j}}\right)^{12}-\left(\frac{\sigma}{r_{i j}}\right)^{6}\right]$

The exact parameters of the force field used in the simulations can be found in the Appendix A.

\subsubsection{Energy minimization}

The energy minimization calculations were made with the METADISE code [21], which implements an energy minimization technique based on classical potential models. The total energy of the system is calculated by summing the interactions between all pairs of atoms up to a cutoff radius of $15 \AA$.
Energy minimization is a method whereby the atomic coordinates iteratively converge toward the atomic configuration with minimum total energy. Since the method finds the nearest local minimum, the result is largely dependent on the initial configuration of the atoms. Thus several initial configurations have to be tested in order to increase the chances of finding a physically meaningful minimum (e.g. different possible cuts for the same surface). Energy minimization cannot describe temperature effects or entropic contributions as other methods such as molecular dynamics simulations can but is computationally less expensive.

For bulk calculations, periodic boundary conditions have been applied in all directions. For surface calculations, as the surface disrupts periodicity in one direction, periodic boundary conditions have been applied in the interface plane only. Along the direction normal to the surface the structure has been divided in two regions, atoms in the region adjacent to the surface were allowed to relax to their minimum energy position whereas atom in the region further away from the interface were fixed at their previously calculated single crystal positions. The depth of the surface region is chosen for complete relaxation of the surface to be possible without restriction due to the fixed region ( $\geq 16.2 \AA$ ). The bulk region has to be deep enough to make long-range coulombic interaction between its top and bottom atoms negligible ( $\geq 80 \AA$ ). This model is valid for grain sizes where an unperturbed bulk structure exists at the core. Several non-polar cuts at different depths along the same surface normal have been calculated, the final structure corresponding to the termination with the lowest energy.

\subsubsection{Molecular dynamics}

The molecular dynamics calculations were made with the dl_poly 2.20 code [22]. Molecular dynamics calculates the time evolution of the position and velocities of the atoms as a function of the forces between the atoms calculated via the atomistic potential. The timestep employed for all simulations was $0.2 \mathrm{fs}$. The simulations were done either at constant volume and constant temperature (NVT, thermostat algorithm employed: Nose-Hoover [23] with a relaxation time of $0.5 \mathrm{ps)}$ or at constant pressure and constant temperature (NPT, barostat-thermostat algorithm employed: Nose-Hoover [23] or Nose-Hoover-Melchionna [24] with a relaxation time of $0.5 \mathrm{ps}$ for both temperature and pressure). The cutoff employed for the short range interactions was $8.5 \AA$, for the coulomb interactions the Ewald summation was employed.

In addition, to be able to estimate the specific heat capacity for the water potential, NPT calculations with varying temperature during the simulation have been done with the LAMMPS code [25,26]. A water box containing 256 molecules was first equilibrated at $\mathrm{T}=300 \mathrm{~K}$ and $0 \mathrm{bar}$ pressure for $0.1 \mathrm{ps}$. Then the temperature was increased linearly from $300 \mathrm{~K}$ to $500 \mathrm{~K}$ during $0.2 \mathrm{~ns}$, then decrease again from $500 \mathrm{~K}$ to $250 \mathrm{~K}$ during $0.3 \mathrm{~ns}$.

The total simulation times will be given in equilibration time plus production run time. For all calculations periodic boundary conditions have been applied in all directions. For bulk portlandite $(6 \times 6 \times 4$ crystallographic units in a, b and c directions), bulk water (2048 water molecules) and portlandite-water solution (box of 2045 water molecules with one calcium ion and two hydroxyl groups) the NPT ensemble was used. For portlandite the simulation box was first equilibrated (NPT simulation with an anisotropic Noose-HooverMelchionna thermostat which allows the cell to relax for $5+15 \mathrm{ps}$ ). Finally the heat of dissolution was calculated as the difference between the configurational energy of the portlandite-water solution $\mathrm{E}_{\text {port_H2O_sol }}$ and the energy of the same number of water molecules in the pure water simulation $\mathrm{N}_{\mathrm{H} 2 \mathrm{O} \text { port_H2O_sol }} / \mathrm{N}_{\mathrm{H} 2 \mathrm{O}, \mathrm{H} 2 \mathrm{O}} * \mathrm{E}_{\mathrm{H} 2 \mathrm{O}}$ minus the energy of one $\mathrm{Ca}(\mathrm{OH})_{2}$ unit in portlandite $1 / \mathrm{N}_{\mathrm{Ca} \text {,port }}{ }^{*} \mathrm{E}_{\text {port }}$ (Eq. 6).

$\Delta H_{\text {sol }}=E_{\text {port_ } \mathrm{H}_{2} \mathrm{O} \_ \text {sol }}-\mathrm{N}_{\mathrm{H}_{2} \mathrm{O}, \text { port_ } \mathrm{H}_{2} \mathrm{O}_{-} \text {sol }} / \mathrm{N}_{\mathrm{H}_{2} \mathrm{O}, \mathrm{H}_{2} \mathrm{O}} \cdot E_{\mathrm{H}_{2} \mathrm{O}}-1 / N_{\text {Ca,port }} \cdot E_{\text {port }}$. 
For portlandite-water interface calculations the simulation box consisted of a portlandite slab delimited on both sides by identical surfaces. On one side of the portlandite slab, a water box has been placed. Perpendicular to the portlandite-water interface the periodic images of the slab are separated by a vacuum gap (Fig. 2) to prevent the development of stresses in the slab. After initial equilibration of the box, the calculations were done at constant volume (NVT). The final simulations were carried out for $10+20$ ps where the first 10 ps were used for equilibration of the system and the following $20 \mathrm{ps}$ were used for all calculations. To calculate the portlandite-water surface energy $\gamma$, the energy of the original portlandite-water slab $E_{\text {port_H2O }}$ was compared to half the energy of a pure portlandite slab $\mathrm{E}_{\text {port,slab }}$ plus half the energy of a pure water slab $\mathrm{E}_{\mathrm{H} 2 \mathrm{O} \text {,slab }}$ both twice the size of the original portlandite or water slab respectively (Eq. (7) and Fig. 2).

$\gamma=1 / A_{\text {surf }} \cdot\left(E_{\text {port_ } H_{2} \mathrm{O}}-0.5 \cdot E_{\text {port }, \text { slab }}-0.5 \cdot E_{\mathrm{H}_{2} \mathrm{O}, \text { slab }}\right)$

\section{Results and discussion}

\subsection{Identification of crystalline phase of formed particles}

XRD patterns of the precipitated samples were all similar to the $\mathrm{XRD}$ pattern of sample $\mathrm{BC}$ presented in Fig. 3. In all cases a crystalline material was obtained, which matched the $\mathrm{Ca}(\mathrm{OH})_{2}$ pattern of portlandite (ICDD 04-0733). It was concluded that a pure material was obtained with this procedure.

\subsection{Carbonation}

Two decomposition steps were observed in the thermogravimetric curves measured on all precipitated samples, as shown with sample B in Fig. 4. The first weight loss occurs around $450^{\circ} \mathrm{C}$ and corresponds to the dehydration of portlandite into calcium oxide $\mathrm{CaO}$. The second weight loss occurs at a higher temperature, around $600{ }^{\circ} \mathrm{C}$, and was attributed to the decomposition of calcium carbonate into $\mathrm{CaO}$. The calculation led to a corresponding amount of 3.5 to $4.3 \mathrm{~mol} \%$ of $\mathrm{CaCO}_{3}$, which may not be visible in X-ray diffractograms.

\subsection{Changes in morphology}

\subsubsection{Qualitative observations}

The SEM images show that particles formed in the presence of either $\mathrm{Cl}^{-}$or $\mathrm{NO}_{3}^{-}$were facetted and regular with three distinct families of facets. The general shape of the particles seems to be the

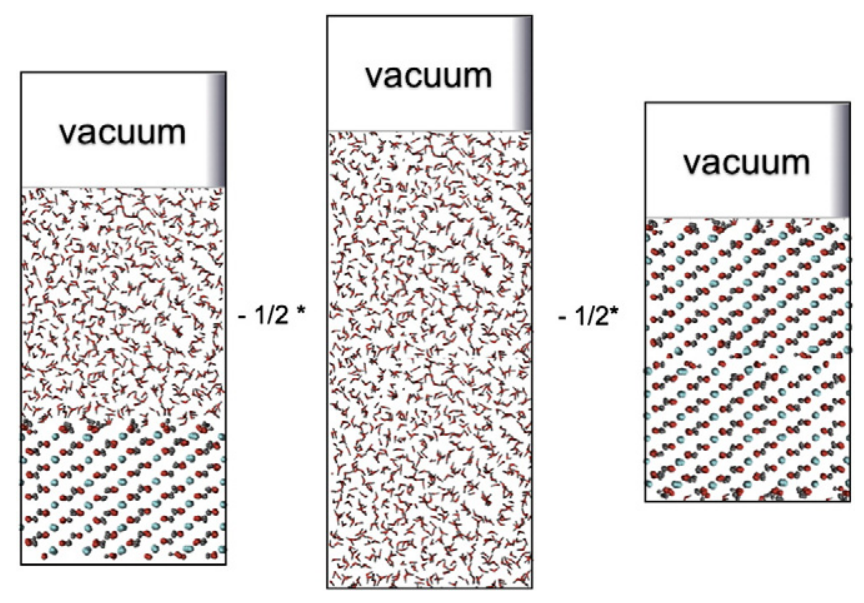

Fig. 2. Schematic view of portlandite-water surface energy calculations.

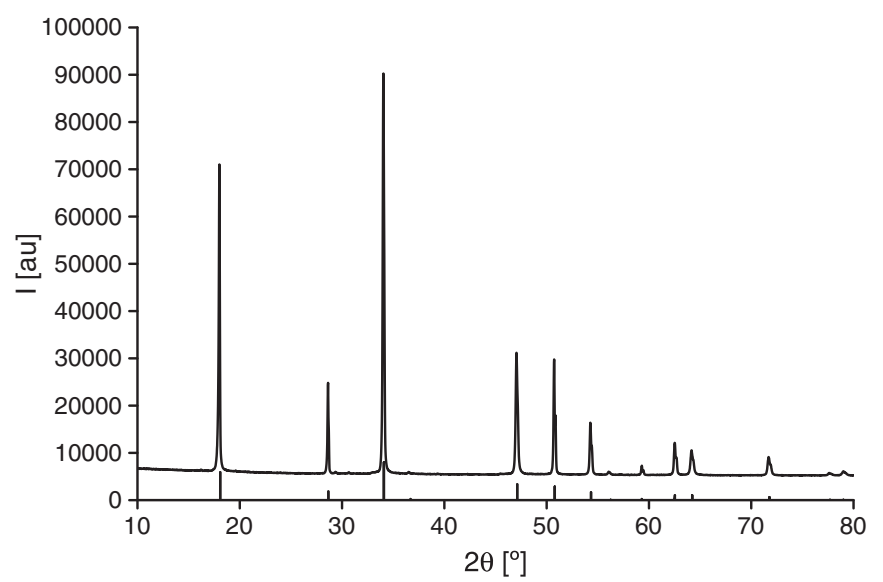

Fig. 3. XRD pattern of a precipitated portlandite (sample BC). Lines correspond to the peaks of ICDD 04-0733 (pure portlandite). Similar patterns were recorded for all samples.

same for the three samples with only either $\mathrm{Cl}^{-}$at 0.2 or $0.3 \mathrm{~mol} / \mathrm{l}$ or $\mathrm{NO}_{3}^{-}$at $0.2 \mathrm{~mol} / \mathrm{l}$ present (samples $\mathrm{B}, \mathrm{BC}$ and $\mathrm{BN}$; see Fig. 5). The particles are slightly elongated in the direction of the [00.1] axis which is clearly discernible because of the trigonal symmetry. The size of the particles for the three samples is similar for all three samples, the median volume diameter of the measured size distributions were between 2.65 and $3.72 \mu \mathrm{m}$ (Table 2), the only trend which might be discernible is a slight decrease in particle size with an increase in chloride concentration from 0.2 to $0.3 \mathrm{~mol} / \mathrm{l}$. Intuitively one could explain such a relation by the adsorption of chloride ions onto portlandite surfaces which might slow down their growth.

Particles formed in the presence of both $0.2 \mathrm{~mol} / \mathrm{l} \mathrm{Cl}^{-}$and $0.2 \mathrm{~mol} / \mathrm{l} \mathrm{SO}_{4}^{2-}$ (sample BS) showed a hexagonal platelet morphology similar to that observed by Harutyunyan et al. [5] for portlandite particles formed by hydration of ASTM Type II Portland cement with $6 \%$ C4A3 $\$$ admixture and in the presence of gypsum. The results are also consistent with results from Berger et al. [27] and Gallucci et al. [4] whose results indicate that the presence of sulfates promotes hexagonal platelet morphology of growing portlandite particles. Since a hexagonal platelet morphology means that particle dimension in [00.1] direction is much lower than in [10.0] direction, these results show that the relative growth speed in [00.1] is reduced by the presence of sulfate ions, either by changing the relative surface energies or by changing the relative growth kinetics. The measured size distribution of the particles is similar to the ones measured in the presence of chlorides or nitrates only (samples B, BC, and BN) with a median volume diameter of $2.60 \mu \mathrm{m}$ (Table 2 ). If the concentration of the sulfate ions is decreased to $0.02 \mathrm{~mol} / \mathrm{l}$ (sample BSO2) the shape of

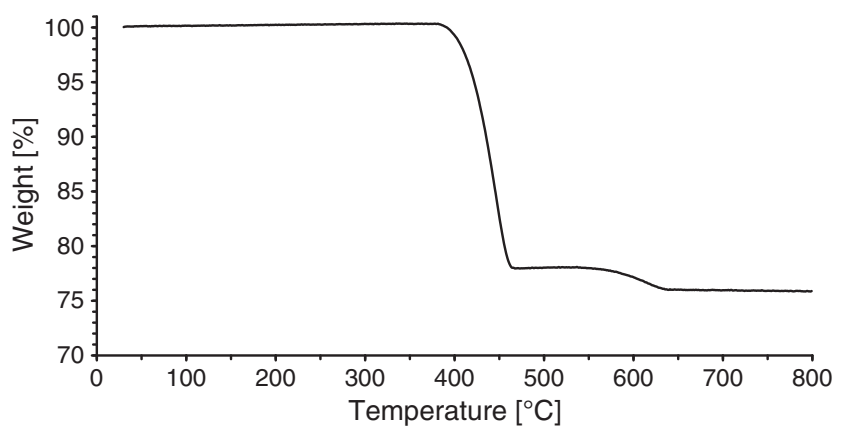

Fig. 4. Thermogravimetric curve obtained for sample B. Heating rate $10{ }^{\circ} \mathrm{C} / \mathrm{min}$ in flowing air $30 \mathrm{ml} / \mathrm{min}$. 
a

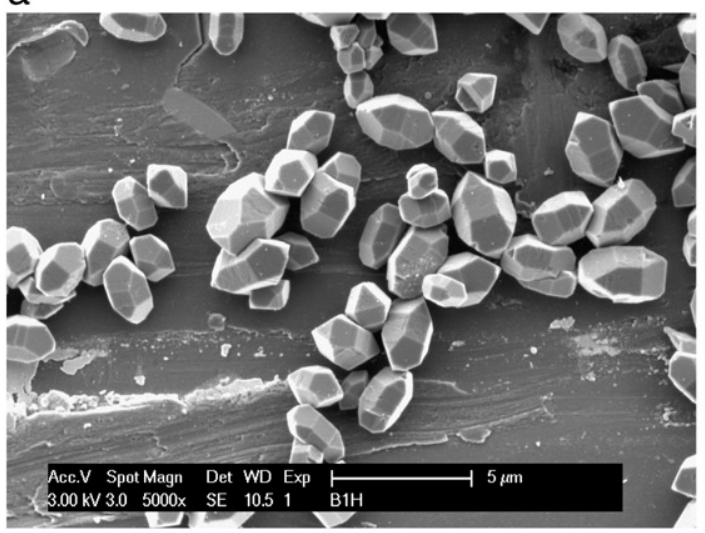

C

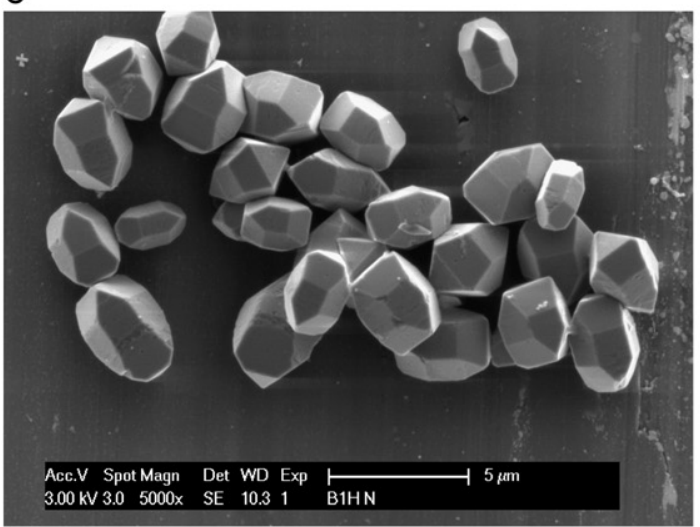

e

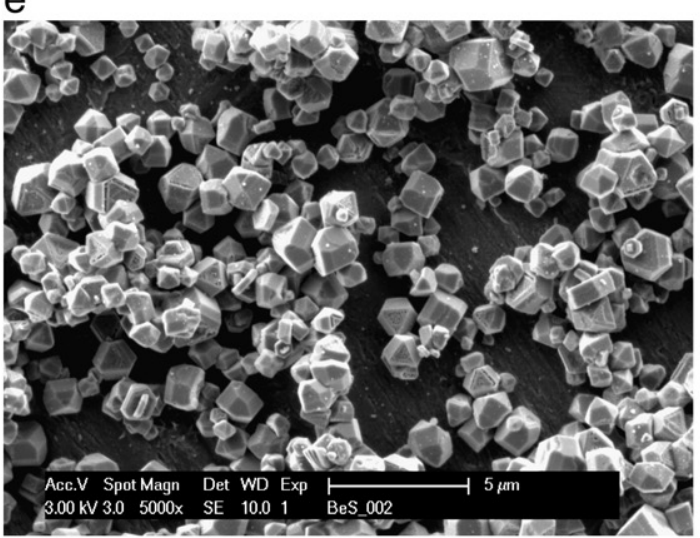

b

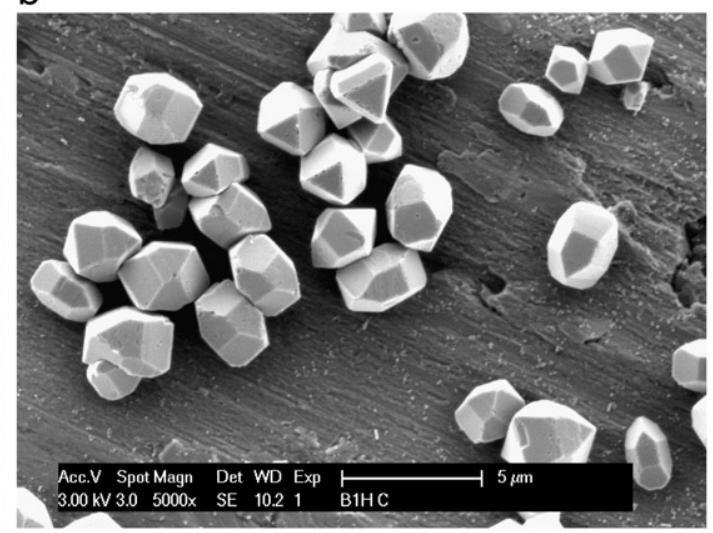

d

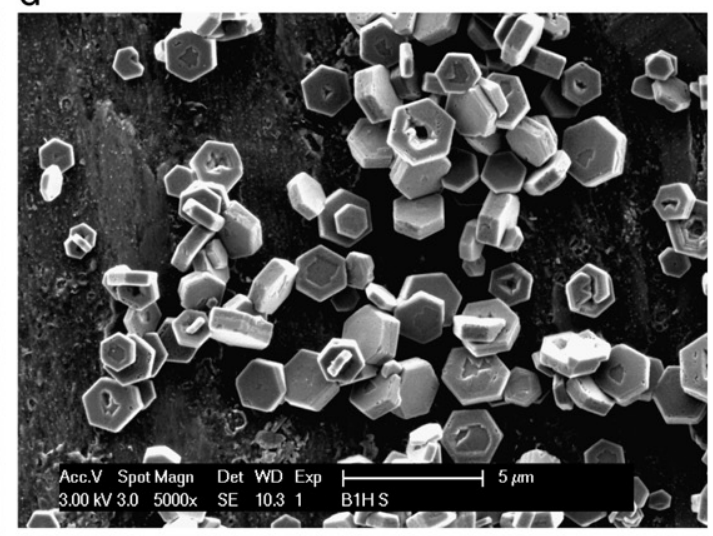

f

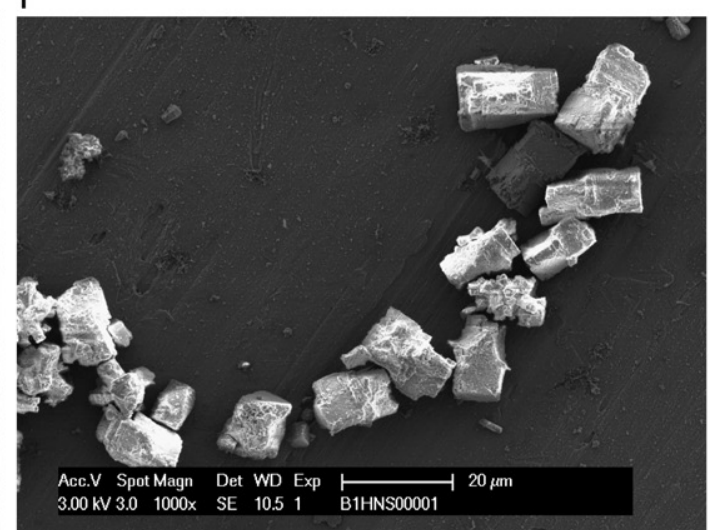

Fig. 5. SEM images of portlandite particles for the different samples. a) B, b) BC, c) BN, d) BS, e) BSO2 and f) BSi.

most particles shows again the three different families of facets observed for samples B, BC and BN. However the particles are no longer elongated in [00.1] direction and some particles of almost

Table 2

Characteristic values of the measured volume size distribution of the particles. $10 \%$ of the measured particles have a size lower than $\mathrm{D}(\mathrm{v} 10), 50 \%$ lower than $\mathrm{D}(\mathrm{v}, 50)$ and $90 \%$ lower than $\mathrm{D}(\mathrm{v}, 90)$.

\begin{tabular}{llll}
\hline & $\mathrm{D}(\mathrm{v}, 10)[\mu \mathrm{m}]$ & $\mathrm{D}(\mathrm{v}, 50)[\mu \mathrm{m}]$ & $\mathrm{D}(\mathrm{v}, 90)[\mu \mathrm{m}]$ \\
\hline B & 0.53 & 3.72 & 7.98 \\
BC & 0.50 & 2.65 & 5.10 \\
BN & 0.50 & 2.93 & 5.60 \\
BS & 0.46 & 2.60 & 5.35 \\
BS02 & 0.43 & 1.85 & 4.26 \\
BSi & Large aggregates about $10 \mu \mathrm{m}$ in size & \\
\hline
\end{tabular}

hexagonal platelet morphology can also be observed, indicating a particle shape in between the ones for sample B without sulfates and for sample BS with a higher concentration of sulfates. The size of the particles however is smaller (median: $1.85 \mu \mathrm{m}$ ) than for both sample $\mathrm{B}$ and sample BS, making it difficult to discern a clear tendency of the particle size with the addition of sulfates.

Finally the addition of $0.001 \mathrm{~mol} / \mathrm{SiO}_{3}^{2-}$ seems to lead to large, irregularly shaped agglomerates of particles. The size of the agglomerates is about $10 \mu \mathrm{m}$. Even though the concentration of silicates was much lower than the concentrations of the other ions, the change in particle morphology is much more marked than for the other samples. This indicates that silicates have a great influence on portlandite morphology and growth. Since silicates are always present in cementitious systems this is of great importance when discussing changes in portlandite morphology in cementitious systems in that the 
influence of some ions might come from the interaction with silicate ions rather than from the interaction with portlandite ions directly and needs to be further explored.

\subsubsection{Quantitative description of facetted regular particles}

The analysis method described in Section 2. made it possible to completely determine the shape of the particles and to clearly identify the facets present ([00.1], [10.0] and [20.3] surfaces). The identification of the low index [00.1] and [10.0] surfaces was expected as these surfaces are part of the hexagonal platelet shape reported as the morphology of naturally found portlandite minerals [28] and of portlandite grown in cementitious systems in the presence of sulfates $[4,5,27]$. The presence of the higher index [20.3] surface was more unexpected.

The quantification of the morphologies indicate that the energy of both the [10.0] and the [20.3] surface increase relative to the [00.1] surface with increasing $\mathrm{Cl}^{-}$concentration (Table 3), the [10.0] surface more so than the [20.3]. Although the change is not very marked and within the spread of the data, the direction of the change is unexpected: The results of Berger and McGregor [27] indicated that the addition of chlorides and nitrates led to an elongation of the particles in the [00.1] direction, indicating an increase of the [00.1] surface energy relative to the [20.3] and [10.0], not the other way around. The apparent inconsistency of our results with the results of Berger and McGregor might be due to several reasons. First of all the particles observed by Berger and McGregor were much larger, of the order of $100 \mu \mathrm{m}$, and irregularly shaped. This means it is possible that they observed aggregates rather than individual particles. Secondly the particles observed by Berger et al. were formed upon the hydration of C3S which means that other ions, namely silicates, were present and had an influence on the shape of the portlandite particles. Thirdly, the effect of the chlorides might change with the concentration. The aspect ratio of the particles observed by Berger and McGregor indicates a relative surface energy of the [10.0] with respect to the [00.1] of $<0.66$, our results show mostly consistent values ( 0.586 for sample B and 0.699 for sample BC).

Replacing the $\mathrm{Cl}^{-}$ions by $\mathrm{NO}_{3}^{-}$ions has a similar effect as increasing the chloride concentration (higher relative surface energies of both the [10.0] and the [20.3] surface), but the effect is more marked. The relative surface energy of the [10.0] surface (0.727) is higher than the value of $<0.66$ reported by Berger and McGregor [27] for particles grown in the presence of nitrates.

The addition of $\mathrm{SO}_{4}^{2-}$ ions leads to an important increase in the energy of the [10.0] surface relative to the [00.1] surface (3.167). The value is consistent with the value of $>2$ reported by Berger and McGregor for particles formed in the presence of sulfates. It is also mostly consistent with the value of 2.5- 2.7 reported by Harutyunyan et al. In fact the consistency of the experimental results of different authors and the current results is remarkable as the systems considered by Berger and McGregor as well as by Harutyunyan et al. contained silicates whereas the current BS samples contained chlorides, both of which are ions which seem to influence the shape of the portlandite particles. It seems that the effect of the sulfate ions dominates the shape of the

Table 3

Relative surface energies measured for samples B, BC, BN and BS as well as calculated via atomistic simulation for particles in water and in vacuum.

\begin{tabular}{|c|c|c|c|c|c|c|c|}
\hline & \multirow[t]{2}{*}{$\begin{array}{l}\text { \# Analyzed } \\
\text { particles }\end{array}$} & \multicolumn{3}{|c|}{$\begin{array}{l}\text { Relative surface } \\
\text { energy }[10.0] /[00.1]\end{array}$} & \multicolumn{3}{|c|}{$\begin{array}{l}\text { Relative surface } \\
\text { energy [20.3]/[00.1] }\end{array}$} \\
\hline & & Mean & Min & Max & Mean & Min & Max \\
\hline B & 9 & 0.586 & 0.499 & 0.625 & 0.660 & 0.595 & 0.716 \\
\hline $\mathrm{BC}$ & 9 & 0.699 & 0.524 & 0.799 & 0.735 & 0.606 & 0.796 \\
\hline $\mathrm{BN}$ & 8 & 0.727 & 0.628 & 0.830 & 0.737 & 0.661 & 0.838 \\
\hline BS & 10 & 3.164 & 2.271 & 4.050 & $>3.011$ & $>2.35$ & $>3.93$ \\
\hline Calc water & - & 1.161 & - & - & 0.705 & - & - \\
\hline Calc vacuum & - & 7.548 & - & - & 12.548 & - & - \\
\hline
\end{tabular}

portlandite particles. Since the [20.3] surface does not appear in the morphology only a lower bound for its relative energy can be calculated. The relative surface energy of the [20.3] surface has to be higher than 3.011 for the facet not to appear in the morphology. This means that the relative energy of both the [00.1] and the [20.3] surfaces increase markedly with the addition of sulfates.

\subsection{Atomistic simulation}

\subsubsection{Performance of employed force field}

For portlandite the overall performance of the force field was very good. The crystallographic unit cell parameters $(a=b=3.59 \AA$, $c=4.90 \AA, \alpha=\beta=90.0^{\circ}, \gamma=120.0^{\circ}$ [1]) are all reproduced with an accuracy of better than $10 \%$ with energy minimization (a: $3.67 \AA$, c: $4.90 \AA, \alpha=\beta=90.0^{\circ}, \gamma=120.0^{\circ}$ ) as well as with molecular dynamics (a: $3.68 \AA$, c: $4.82 \AA, \alpha=90.1^{\circ}, \beta=90.0^{\circ}, \gamma=120.0^{\circ}$ ). The Voigt elastic and shear modulus calculated with energy minimization ( $K_{\text {Voigt: }}: 40.07 \mathrm{GPa} ; \mathrm{G}_{\text {Voigt }}: 23.03 \mathrm{GPa}$ ) seem to be consistent with the experimentally observed values (Koigt: $46.93 \mathrm{GPa}$; $\mathrm{G}_{\text {Voigt: }}$ 19.29 GPa). The Reuss modulus however seems to be underestimated (K $K_{\text {Reuss }}$ : $19.70 \mathrm{GPa} ; \mathrm{G}_{\text {Reuss }}$ : $5.26 \mathrm{GPa}$ ) compared to experimentally observed values ( $K_{\text {Reuss }}$ : $32.39 \mathrm{GPa}$; $\mathrm{G}_{\text {Reuss }}$ : $13.39 \mathrm{GPa}$ ).

The water description is a definite strength of the employed force field. The complex phase diagram of water is reproduced with very good accuracy with only a slight shift in temperature [20]. The experimentally observed radial distribution function [29], describing the spatial distribution of the water molecules, is very well reproduced (Fig. 6). In addition thermodynamic properties such as heat of vaporization $(11.30 \mathrm{~kJ} / \mathrm{mol})$, specific heat capacity $(85.1 \mathrm{~kJ} / \mathrm{mol} * \mathrm{~K})$ and the self diffusion constant $\left(1.91 \mathrm{~m}^{2} / \mathrm{s}\right)$ are consistent with experimentally observed values ( $\mathrm{H}_{\text {vap }}$ : $10.52 \mathrm{~kJ} / \mathrm{mol}$; Cp: $75.6 \mathrm{~kJ} / \mathrm{mol} * \mathrm{~K} ; \mathrm{D}: 2.3 \mathrm{~m}^{2} / \mathrm{s}$ [20]).

By placing one Ca ion and two hydroxyl ions in a box of water a portlandite solution has been simulated. The calculated heat of solution (Eq. (6)) was $161 \mathrm{~kJ} / \mathrm{mol}$. This value is in good agreement with the value of $134-135 \mathrm{~kJ} / \mathrm{mol}$ reported in literature [30,31]. To further validate the results, the radial distribution of water molecules around the Ca and hydroxyl ion was compared to values reported in literature by Fulton et al. [32] and by Botti et al. [33]. The calcium ion environment is very close to that reported by Fulton et al. The calcium-water oxygen nearest neighbor distance calculated here is $2.47 \AA$ and for calcium-water hydrogen $3.08 \AA$. (experimental

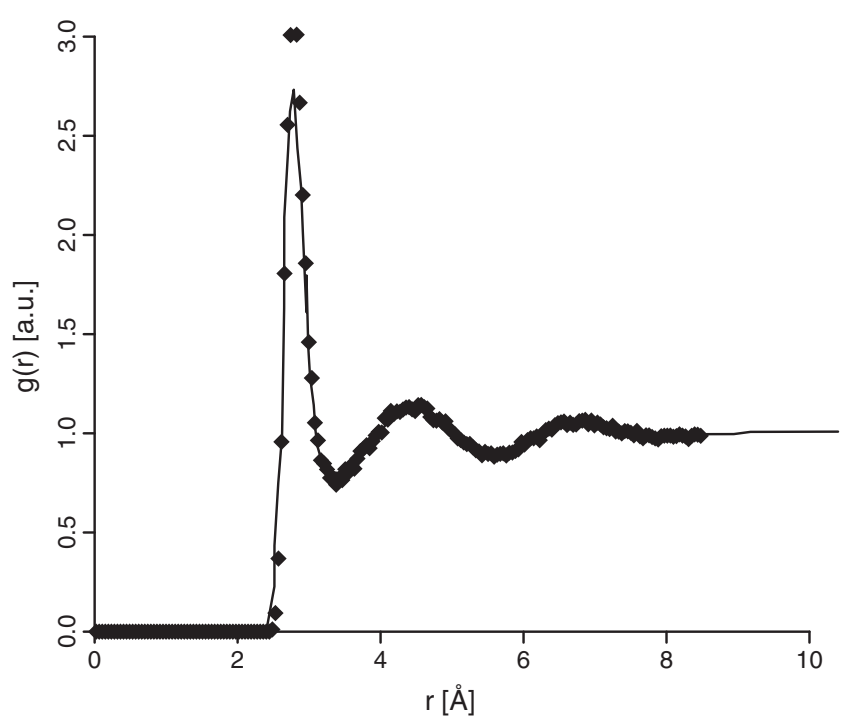

Fig. 6. Radial distribution function of $\mathrm{O}-\mathrm{O}$ in water. Line: experimental results reported by Soper et al. [26]. Diamonds: current results. 
observations by Fulton et al. Ca-O(water): $2.44 \AA$, Ca-H(water): $2.97 \AA$ ). The calcium ion seems to be coordinated by about 7.6 water molecules, again this compares well with the previously reported value of $7.2+/-1.2$. The chemical environment of the hydroxyl ion is slightly less consistent with experimental observations reported by Botti et al. The nearest neighbor distances ( $\mathrm{O}$ (hydroxyl)-H(water): $1.7 \AA$; $\mathrm{H}$ (hydroxyl)-O(water): $2.73 \AA$ ) are higher than the ones reported in literature ( $\mathrm{O}$ (hydroxyl)-H(water): $1.4 \AA$; $\mathrm{H}$ (hydroxyl)$\mathrm{O}$ (water): $2.59 \AA$ ). Probably as a consequence of the higher nearest neighbor distances the number of nearest neighbor water molecules (O(hydroxyl): 6 water molecules; $\mathrm{H}$ (hydroxyl)-O(water): 8 water molecules) is also slightly higher than previously reported values (O(hydroxyl): 4 water molecules; H(hydroxyl): 6 water molecules). However the general shape of the hydroxyl-water radial distribution function is well reproduced (Fig. 7). Additionally, as Chen et al. pointed out the experimentally measured nearest neighbor peak of the radial distribution function might in fact be a superposition of "true" nearest neighbor water and of $\mathrm{H}-\mathrm{O} \cdots \mathrm{H}^{\cdots \cdots} \mathrm{O}-\mathrm{H}$ pairs which come from proton exchange events [34].

Further discussion of the force field and a comparison with other force fields commonly used for cements can be found in [14].

\subsubsection{Simulation of portlandite surfaces}

Atomistic simulation was used to calculate the surface energies for both portlandite in vacuum and in water. The calculation of the energy in vacuum is straightforward as there is no water and hence dynamics play only a subordinate role. The portlandite-vacuum surface energies are hence calculated by energy minimization. The resulting surface energies are: [00.1]: $0.073 \mathrm{~J} / \mathrm{m}^{2}$, [10.0]: $0.551 \mathrm{~J} / \mathrm{m}^{2}$, [20.3]: $0.916 \mathrm{~J} / \mathrm{m}^{2}$. These energies result in a hexagonal platelet shape of the equilibrium morphology with an aspect ratio of 7.5 (Fig. 8). This is consistent with the Handbook of Mineralogy [28] which reports a hexagonal platelet morphology of portlandite and perfect cleavage along the [00.1] surface indicating a low [00.1] surface energy. In vacuum the [20.3] surface seems to have a very high energy, in fact the energy is more then ten times the energy of the [00.1] surface and almost twice the energy of the [10.0] surface.

The portlandite-water surface energies calculated by molecular dynamics are: [00.1]: $0.110 \mathrm{~J} / \mathrm{m}^{2},[10.0]: 0.128 \mathrm{~J} / \mathrm{m}^{2},[20.3]: 0.078 \mathrm{~J} / \mathrm{m}^{2}$. All surface energies are slightly lowered by the presence of water, indicating a hydrophilic character. The surface energy of the [00.1] surface however, which has an already very low energy in vacuum,

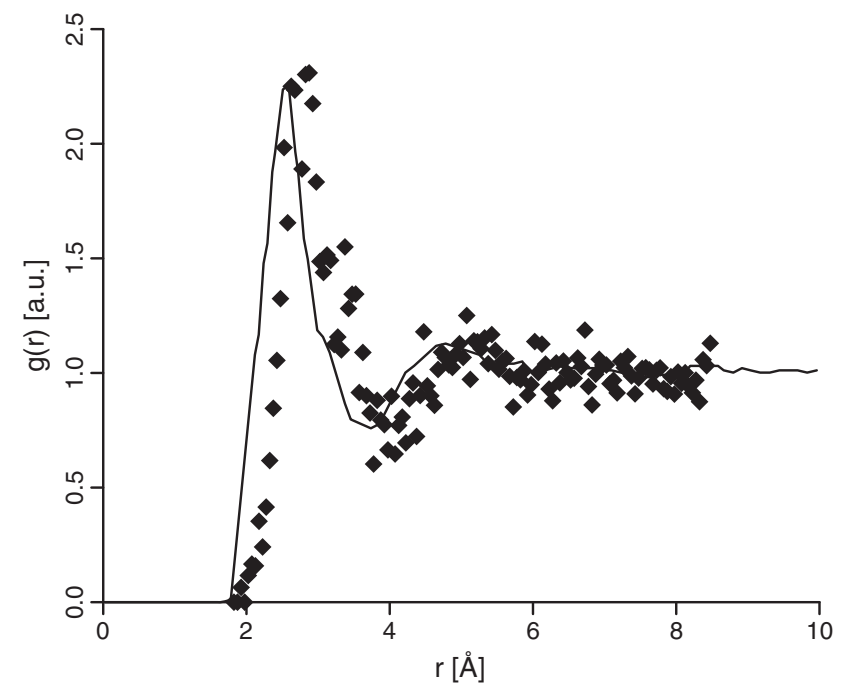

Fig. 7. Radial distribution function of water hydrogen atoms around the hydroxyl oxygen atoms in aqueous solution. Line: experimental results reported by Botti et al. [30]. Diamonds: current results.

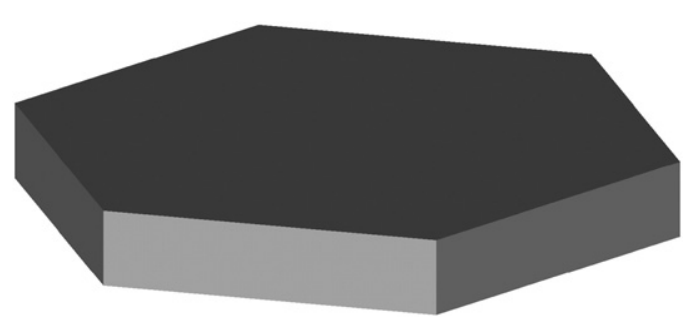

Fig. 8. Calculated equilibrium morphology of portlandite in vacuum. Dark gray: [00.1] facet, light gray: [10.0] surface.

seems to be influenced only slightly by the presence of water. The surface energies of both the [10.0] surface and the [20.3] surface was reduced noticeably by the presence of water but the effect is much more marked for the [20.3] surface. In fact the [20.3] surface which has is a high energy surface in vacuum and does not appear in the portlandite-vacuum morphology has a similar energy to the [00.1] and the [10.0] surface in water and becomes part of the calculated portlandite-water equilibrium morphology (Fig. 9). In fact the calculated differences between the surface energies are very small and are probably smaller than the resolution of the calculation method. These results explain the observation of [20.3] surfaces for most of the precipitated facetted portlandite particles. The mean surface energy of the calculated equilibrium morphology is $0.081 \mathrm{~J} / \mathrm{m}^{2}$, which is consistent with the portlandite-water surface energy estimation of $0.114 \mathrm{~J} / \mathrm{m}^{2}$ of Harutyunyan et al. [5]. The relative surface energies of both the [10.0] and the [20.3] surfaces with respect to the [00.1] surface are similar to the experimentally observed relative surface energies for samples $\mathrm{B}, \mathrm{BC}$ and $\mathrm{BN}$ indicating that $\mathrm{Cl}^{-}$and $\mathrm{NO}_{3}^{-}$ ions might only have a very small effect on the equilibrium morphology (Table 3). Sulfates on the other hand seems to increase the relative energies of both the [10.0] and the [20.3] surface. The next step in the atomistic simulations is to make the link with the effect of the different ions in solution, by incorporating nitrate, chloride and sulfate ions and observe their influence on the interfacial energies.

\subsubsection{Comparison to other results in literature}

Previous simulation work on portlandite-water interfaces is scarce, however Kalinichev et al. did look at the [00.1] surface of portlandite in water. The authors focused, among other things, on the structure of water above the portlandite surface. In order to be able to compare our result to theirs an additional simulation of the [00.1]

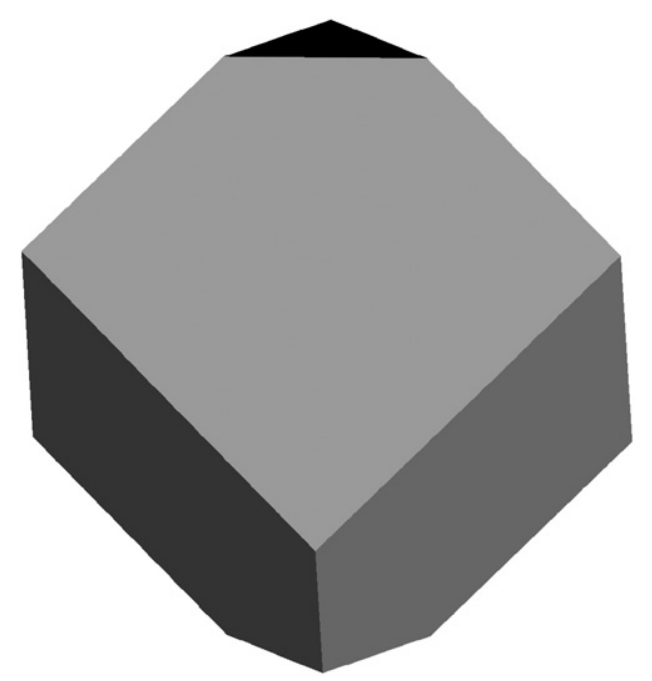

Fig. 9. Calculated equilibrium morphology of portlandite in water. Dark gray: [00.1] facet, light gray: [20.3] facet. 
surface was carried out with a timestep of $0.7 \mathrm{fs}$ and a total simulation length, after equilibration, of $84 \mathrm{ps}$. These conditions are similar to the ones employed by Kalinichev et al. The density profile of the water hydrogen and the water oxygen over the surface was then calculated (Fig. 10). By comparing the density profile with the one reported in [9] both similarities and differences can be distinguished. The positions of the peaks corresponding to the first water layer are similar for both profiles. The position of the first and second hydrogen peak for our results is $1.6 \AA$ and $3.3 \AA$ respectively and those reported by Kalinichev et al. are at $\sim 1.7 \AA$ and $\sim 3.1 \AA$ [9]. The first peak of the oxygen density profile is 2.5-3.5 Å whereas Kalinichev et al. reported the oxygen position of the first water layer to be 2.3-2.6 [10]. The form and magnitude of the peaks are however slightly different from the results reported by Kalinichev et al.: the first hydrogen peak is of lesser magnitude and the first oxygen peak is broader than observed by Kalinichev et al. Without doing a complete hydrogen bonding analysis these results indicate that we have fewer water molecules with a hydrogen pointing toward the surface in our simulation and a more diffuse first water layer. Probably as a consequence of the more diffuse first water layer the second water layer is farther from the surface compared to the results of Kalinichev et al. A comparison between the two force fields reported in [14] might give some indications as to the source of the observed differences.

\section{Conclusion}

The morphology of portlandite particles formed by coprecipitation in the presence of different ions has been observed. In the presence of either chloride or nitrate ions, the portlandite particles formed have a regular and facetted shape and have sizes between 2.7 and $3.7 \mu \mathrm{m}$. The facets present have been identified as [00.1], [10.0] and [20.3] facets. The relative surface energy of the [10.0] surface with respect to the [00.1] surface and the aspect ratio of the particles was 0.59-0.73.

The addition of sulfates leads to the formation of particles with a hexagonal platelet shape. In terms of relative surface energies this is equivalent to raising the relative surface energy of both the [10.0] and the [20.3] surface energy with respect to the [00.1] surface. The observed [10.0] relative surface energy and thus the aspect ratio of the particles was 3.16. The size of the particles $(2.6 \mu \mathrm{m})$ did not seem to have been influenced greatly by the presence of the sulfate ions.

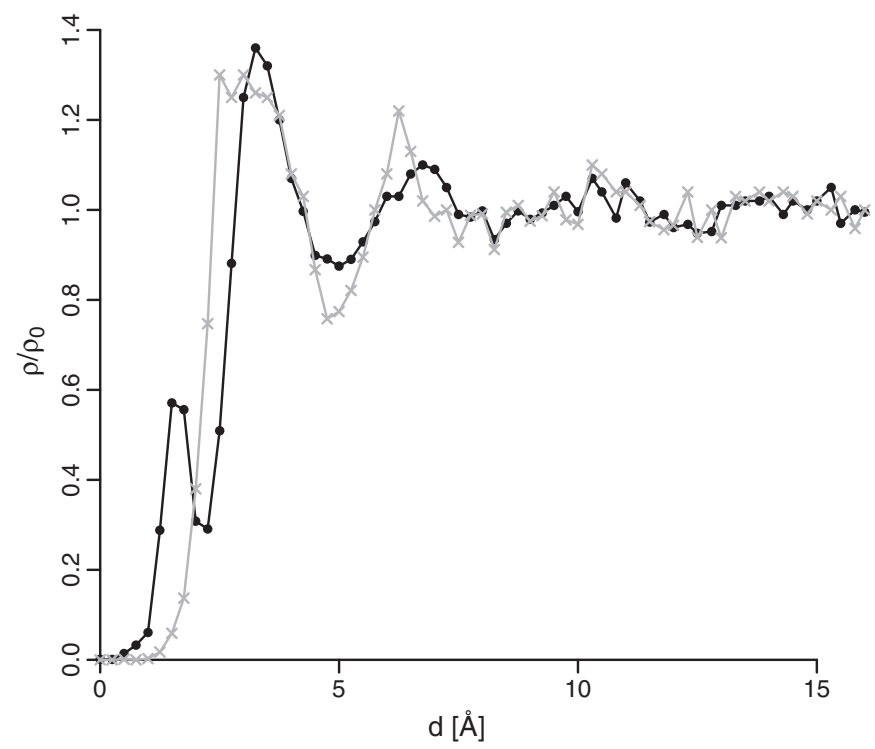

Fig. 10. Density profile of the number density of water hydrogen (black line) and water oxygen (gray line) relative to the bulk density at the [00.1] surface of portlandite. $d$ is the distance from the portlandite oxygen layer closest to the surface.
The addition of silicates leads to large agglomerates $(\sim 10 \mu \mathrm{m})$ which are irregularly shaped. As the concentration of the silicate ions was much smaller than the concentration of the other ions, their effect upon the growth of portlandite particles seems to be the most marked.

Atomistic simulations showed that the [20.3] surface, which has a very large energy in vacuum, is greatly stabilized by the presence of water, explaining the appearance of [20.3] facets for most experimentally observed particles. The energy of the [00.1] surface on the other hand, which has an already very low surface energy in vacuum, is only influenced very slightly. Further simulations on the effects of the chloride, nitrate and sulfate ions will help further elucidate the mechanisms behind the modification of morphologies observed.

\section{Acknowledgments}

This work was financially supported by the industrial-academic research network on cement and concrete, nanocem. The authors would like to thank Robert Flatt, Ellis Gartner, Patrick Juilland, Steve Parker and Karen Scrivener for fruitful discussions.

\section{Appendix A. Supplementary data}

Supplementary data to this article can be found online at doi:10.1016/ j.cemconres.2011.04.009.

\section{References}

[1] H.E. Petch, The hydrogen positions in portlandite, $\mathrm{Ca}(\mathrm{OH}) 2$, as indicated by the electron distribution, Acta Crystallogr. 14 (1961) 950-957.

[2] L. Desgranges, D. Grebille, G. Calvarin, G. Chevrier, N. Floquet, J. Niepce, Hydrogen thermal motion in calcium hydroxide: $\mathrm{Ca}(\mathrm{OH}) 2$, Acta Crystallogr. B Struct. Sci. 49 (1993) 812-817.

[3] I.G. Richardson, Tobermorite/jennite- and tobermorite/calcium hydroxide-based models for the structure of $\mathrm{C}-\mathrm{S}-\mathrm{H}$ : applicability to hardened pastes of tricalcium silicate, [beta]-dicalcium silicate, Portland cement, and blends of Portland cement with blast-furnace slag, metakaolin, or silica fume, Cem. Concr. Res. 34 (2004) 1733-1777.

[4] E. Gallucci, K. Scrivener, Crystallisation of calcium hydroxide in early age model and ordinary cementitious systems, Cem. Concr. Res. 37 (2007) 492-501.

[5] V. Harutyunyan, A. Kirchheim, P. Monteiro, A. Aivazyan, P. Fischer, Investigation of early growth of calcium hydroxide crystals in cement solution by soft X-ray transmission microscopy, J. Mater. Sci. 44 (2009) 962-969.

[6] C.L. Freeman, J.H. Harding, D.J. Cooke, J.A. Elliott, J.S. Lardge, D.M. Duffy, New forcefields for modeling biomineralization processes, J. Phys. Chem. C 111 (2007) 11943-11951.

[7] S. Kerisit, S.C. Parker, J.H. Harding, Atomistic simulation of the dissociative adsorption of water on calcite surfaces, J. Phys. Chem. B 107 (2003) 7676-7682.

[8] R.T. Cygan, J.J. Liang, A.G. Kalinichev, Molecular models of hydroxide, oxyhydroxide, and clay phases and the development of a general force field, Journal of Physical Chemistry B-Condensed Phase 108 (2004) 1255-1266.

[9] A.G. Kalinichev, R.J. Kirkpatrick, Molecular dynamics modeling of chloride binding to the surfaces of calcium hydroxide, hydrated calcium aluminate, and calcium silicate phases, Chemistry of Materials 14 (2002) 3539-3549.

[10] A.G. Kalinichev, J. Wang, R.J. Kirkpatrick, Molecular dynamics modeling of the structure, dynamics and energetics of mineral-water interfaces: application to cement materials, Cement and Concrete Research 37 (2007) 337-347.

[11] A.R. Roosen, R.P. McCormack, W.C. Carter, Wulffman: a tool for the calculation and display of crystal shapes, Comput. Mater. Sci. 11 (1998) 16-26.

[12] J.A. Nelder, R. Mead, A simplex method for function minimization, Comput. J. 7 (1965) 308-313.

[13] M. Galassi, GNU Scientific Library Reference Manual, 3rd ed, 2009.

[14] S. Galmarini, S.C. Parker, P. Bowen, Atomistic Simulation of Portlandite, Proc.13th ICCC, Madrid in press. (2011).

[15] G.V. Lewis, C.R.A. Catlow, Potential models for ionic oxides, J. Phys. Chem. C 18 (1985) 1149-1161.

[16] A. Pavese, M. Catti, G.D. Price, R.A. Jackson, Interatomic potentials for CaCO 3 polymorphs (calcite and aragonite), fitted to elastic and vibrational data, Phys. Chem. Miner. 19 (1992) 80-87.

[17] S. Kerisit, D.J. Cooke, D. Spagnoli, S.C. Parker, Molecular dynamics simulations of the interactions between water and inorganic solids, J. Mater. Chem. 15 (2005) 1454-1462.

[18] J.P. Allen, W. Gren, M. Molinari, C. Arrouvel, F. Maglia, S.C. Parker, Atomistic modelling of adsorption and segregation at inorganic solid interfaces, Mol. Simul. 35 (2009) 584-608.

[19] U. Aschauer, P. Bowen, S. Parker, Oxygen vacancy diffusion in alumina: new atomistic simulation methods applied to an old problem, Acta Mater. 57 (2009) 4765-4772. 
[20] J.L.F. Abascal, C. Vega, A general purpose model for the condensed phases of water: TIP4P/2005, J. Chem. Phys. 123 (2005) 234505-234512.

[21] G.W. Watson, E.T. Kelsey, N.H. de Leeuw, D.J. Harris, S.C. Parker, Atomistic simulation of dislocations, surfaces and interfaces in $\mathrm{MgO}$, J. Chem. Soc., Faraday Trans. 92 (1996) 433.

[22] W. Smith, T.R. Forester, DL_POLY_2.0: a general-purpose parallel molecular dynamics simulation package, J. Mol. Graphics 14 (1996) 136-141.

[23] S. Nosé, An extension of the canonical ensemble molecular dynamics method, Mol. Physiol. 57 (1986) 187-191.

[24] S. Melchionna, Constrained systems and statistical distribution, Phys. Rev. E 61 (2000) 6165.

[25] S. Plimpton, Fast parallel algorithms for short-range molecular dynamics, J. Comput. Phys. 117 (1995) 1-19.

[26] S. Plimpton, R. Pollock, M. Stevens, Particle-mesh Ewald and rRESPA for parallel molecular dynamics simulations, Proceedings of the Eighth Siam Conference on Parallel Processing for Scientific Computing, 1997.

[27] R. Berger, J. McGregor, Influence of admixtures on the morphology of calcium hydroxide formed during tricalcium silicate hydration, Cem. Concr. Res. 2 (1972) 43-55.
[28] J.W. Anthony, R.A. Bideaux, K.W. Bladh, M.C. Nichols, Handbook of Mineralogy, Halides, Hydroxides, Oxides, Vol. III, Mineral data publishing, 1997.

[29] A.K. Soper, The radial distribution functions of water and ice from 220 to $673 \mathrm{~K}$ and at pressures up to $400 \mathrm{MPa}$, Chem. Phys. 258 (2000) 121-137.

[30] B. Lothenbach, F. Winnefeld, Thermodynamic modelling of the hydration of Portland cement, Cem. Concr. Res. 36 (2006) 209-226.

[31] J.S. Seewald, W.E. Seyfried Jr., Experimental determination of portlandite solubility in $\mathrm{H} 20$ and acetate solutions at $100-350{ }^{\circ} \mathrm{C}$ and 500 bars: constraints on calcium hydroxide and calcium acetate complex stability, Geochim. Cosmochim. Acta 55 (1991) 659-669.

[32] J.L. Fulton, S.M. Heald, Y.S. Badyal, J.M. Simonson, Understanding the effects of concentration on the solvation structure of $\mathrm{Ca} 2+$ in aqueous solution. I: the perspective on local structure from EXAFS and XANES, J. Phys. Chem. A 107 (2003) 4688-4696.

[33] A. Botti, F. Bruni, S. Imberti, M.A. Ricci, A.K. Soper, Solvation of hydroxyl ions in water, J. Chem. Phys. 119 (2003) 5001.

[34] B. Chen, I. Ivanov, J.M. Park, M. Parrinello, M.L. Klein, Solvation structure and mobility mechanism of $\mathrm{OH}-$ : a Car-Parrinello molecular dynamics investigation of alkaline solutions, J. Phys. Chem. B 106 (2002) 12006-12016. 